\title{
A Galaxy in VHE Gamma-rays: Observations of the Galactic Plane with the H.E.S.S. array
}

\author{
R.D. Parsons* \\ Max-Planck-Institut für Kernphysik, Heidelberg, Germany \\ E-mail: daniel.parsons@mpi-hd.mpg.de \\ P. Bordas
}

Max-Planck-Institut für Kernphysik, Heidelberg, Germany

\section{S. Klepser}

DESY, Zeuthen, Germany

\section{on behalf of the H.E.S.S. Collaboration}

The H.E.S.S. gamma-ray observatory has opened a window on our Galaxy at very high energies (VHEs) with resolutions and sensitivities never before possible, discovering a rich zoo of source classes as well as a number of yet to be identified VHE emitters.

The H.E.S.S. data set obtained after more than 14 years of observations, together with the advanced data analysis techniques developed in the last years, has allowed us to study the Galactic Centre region with unprecedented detail, to image supernova remnant shells with an exceptional precision and identify new shell candidates from VHE observations alone, to reveal the extreme properties of $\mathrm{TeV}$ emitting pulsar wind nebulae, to unveil the variable emission from pulsars and gamma-ray binaries, and to characterise the large-scale diffuse emission from the galactic plane. In this contribution we will present a summary of the new H.E.S.S. results on galactic sources, soon to be published as a Special Issue in Astronomy and Astrophysics, as well as the latest observations made by the second phase of the H.E.S.S. experiment, extending the energy range below $100 \mathrm{GeV}$ and providing an excellent cross-over with the energy range seen by the Fermi satellite.

35th International Cosmic Ray Conference - ICRC2017

10-20 July, 2017

Bexco, Busan, Korea

${ }^{*}$ Speaker. 


\section{Introduction}

The H.E.S.S. telescope array is an array of 5 imaging atmospheric Cherenkov telescopes, placed at an altitude of $1800 \mathrm{~m}$ in the Khomas highlands of Namibia. Its southern hemisphere site makes it perfectly positioned for observations of the Milky Way, therefore a significant fraction of H.E.S.S. observing time over the last 14 years of observation has been spent observing the galactic plane.

\section{The H.E.S.S. Galactic Plane Survey}

The culmination of the H.E.S.S. galactic plane survey is the H.E.S.S. Galactic Plane Survey (HGPS) [1]. This survey of the visible galactic plane from the H.E.S.S. location comprises of almost 2700 hours of observations and has a flux sensitivity of better than $2 \%$ along the galactic plane. Figure 1 shows the flux maps of the HGPS region. Additionally an automated source fitting procedure was applied to the HGPS region, which in total identified 78 sources.

Such high sensitivity maps and the source catalogue allow never before possible studies of source populations (for example the population study of pulsar wind nebula candidates in the galaxy [2]), as well as in depth studies individual source regions (for example the detailed morphological and spectral studies of the supernova remnant Vela Jr [3]).

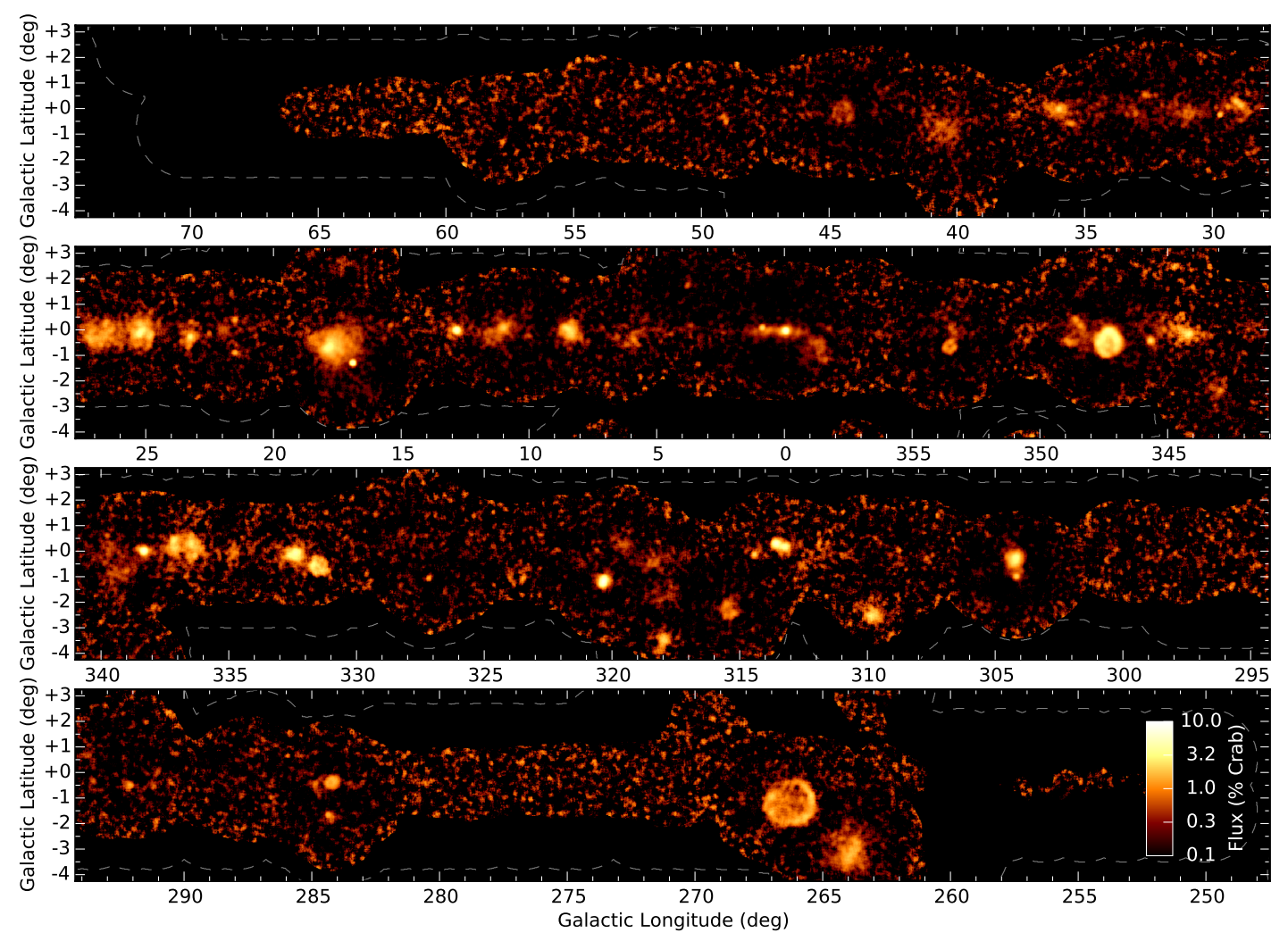

Figure 1: Integral flux of the HGPS region above $1 \mathrm{TeV}$ using a correlation radius of $0.1^{\circ}$ and assuming spectral index 2.3, in units of \% Crab flux. Images reproduced from [1]. 


\section{New Gamma-ray Shells in the HGPS}

One study made possible by the by the HGPS was the blind search for supernova remnant (SNR) shells in the gamma-ray data [4]. Such a search was performed and discovered 3 previously unknown SNR shell candidates (figure 2), HESS J1534-571, HESS J1614-518 and HESS J1912+101. Of these 3 SNR candidates only one (HESS J1534-571) has a resolved shell at other wavelengths.
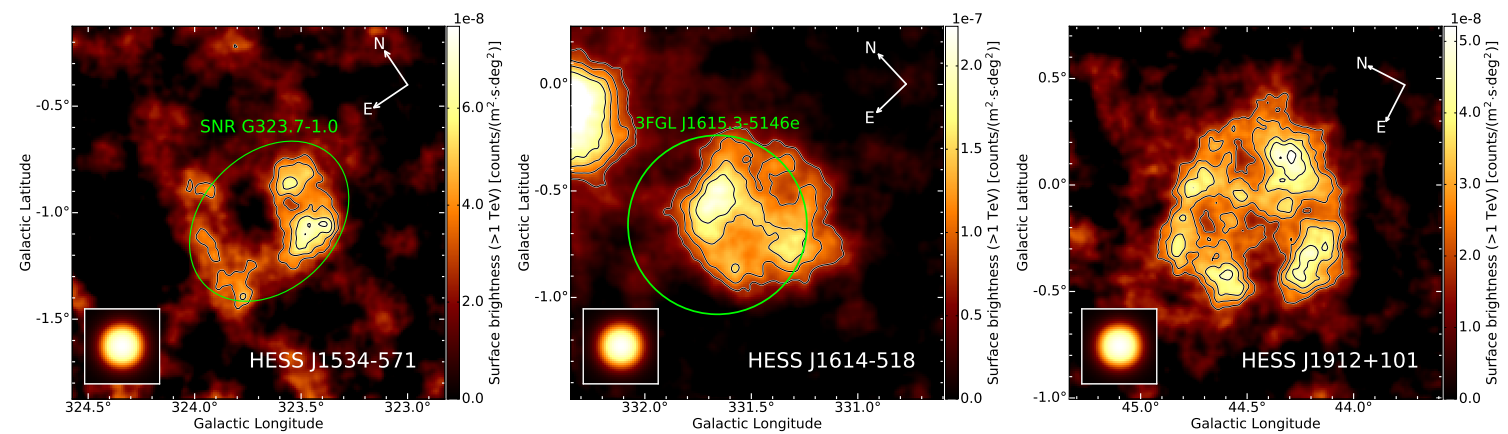

Figure 2: TeV surface brightness map of shell candidate derived with H.E.S.S.. Green ellipses show the extent of associated sources seen at other wavelengths. Images reproduced from [4].

\section{RX J1713-3946: Emission Beyond the X-ray Shell}

The shell-type supernova remnant RX J1713.7-3946 is and old friend to the H.E.S.S. collaboration, with 3 H.E.S.S. papers being published on this source in the past. However, the extremely deep observations and improved analysis techniques now available to H.E.S.S. allowed the collaboration to perform a spectral and morphological analysis of the remnant with unprecedented detail [5]. One possible study was the comparison of the size of the SNR as seen in X-rays with its size seen in gamma rays, shown in figure 3 (right). The comparison of the two profiles shows a clear increase in the size of the SNR in the gamma-ray maps, which is an indication of particle acceleration and escape at the shock front of the SNR.

\section{The Galactic Centre}

In order to further investigate the Galactic Centre ridge emission the H.E.S.S. collaboration recently performed a study of the diffuse gamma-ray emission in the galactic ridge [6]. Using the deep dataset H.E.S.S. has taken on the galactic centre, combined with high resolution analysis techniques, a likelihood fitting technique can be used to extract an empirical model of gamma-ray emission in the galactic centre region. Initially only the two point sources (HESS J1745-290 and $\mathrm{G}+0.9$ ) are included in the fit and further components are successively added to improve the fit. The ultimate result is a multi-component model which well describes emission along the galactic ridge (Figure 4).

Importantly an additional point-like source was required in the fitting process, discovered close to a arc-like feature seen in radio emission (the "arc source"). The position of this source shows good coincidence with the pulsar wind nebula G0.13-0.11. 

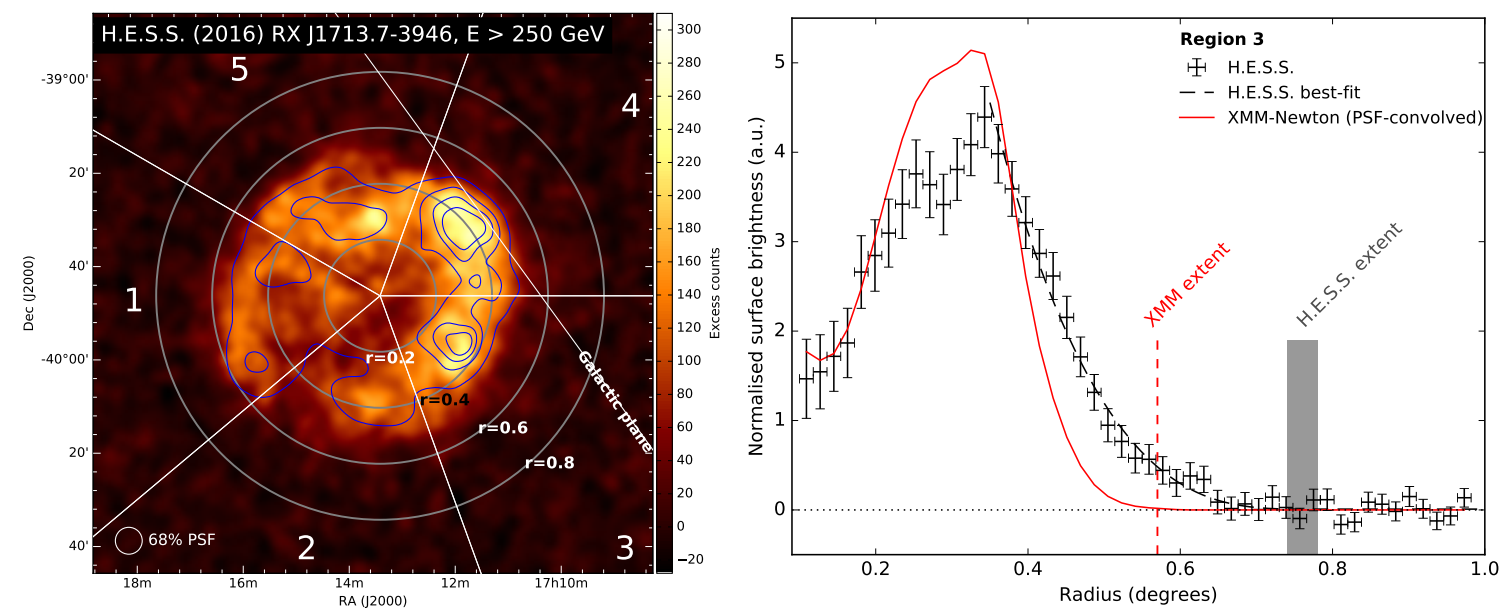

Figure 3: Left: Map of excess gamma-like events in the RX J1713.7-3946 region as seen by H.E.S.S.. Contours show the X-ray image seen by XMM-Newton. Right: Gamma-ray and X-ray profiles seen for region 3 marked on the left plot. Images reproduced from [5].

Using the data mentioned previously it was also possible to make an in depth analysis of the nature of the galactic ridge emission [7] and extract the properties of the accelerator injection protons into this region. The profile of the gamma-ray excess along the galactic plane was extracted (figure 5) and shows a $\frac{1}{r}$ dependence on distance from the galactic centre, which indicates that the central injector has continuously injected protons for around $10^{4}$ years and the cosmic-rays propagate by diffusion [7].

Additionally the gamma-ray spectrum of the diffuse emission was extracted from the region surrounding the central gamma-ray source (shown in figure 5 ). The gamma-ray spectrum has a spectral index of 2.3 and extends to an energy of $50 \mathrm{TeV}$ with no significant spectral cut-off. The gamma-ray spectrum was modelled by solving the proton transport equations and results in a proton injection spectrum extending to $\mathrm{PeV}$ energies, the first detection of a galactic PeVatron. For more information on this study see [7].

\section{Acknowlegements}

The support of the Namibian authorities and of the University of Namibia in facilitating the construction and operation of H.E.S.S. is gratefully acknowledged, as is the support by the German Ministry for Education and Research (BMBF), the Max Planck Society, the German Research Foundation (DFG), the Alexander von Humboldt Foundation, the Deutsche Forschungsgemeinschaft, the French Ministry for Research, the CNRS-IN2P3 and the Astroparticle Interdisciplinary Programme of the CNRS, the U.K. Science and Technology Facilities Council (STFC), the IPNP of the Charles University, the Czech Science Foundation, the Polish National Science Centre, the South African Department of Science and Technology and National Research Foundation, the University of Namibia, the National Commission on Research, Science \& Technology of Namibia (NCRST), the Innsbruck University, the Austrian Science Fund (FWF), and the Austrian Federal Ministry for Science, Research and Economy, the University of Adelaide and the Australian Re- 

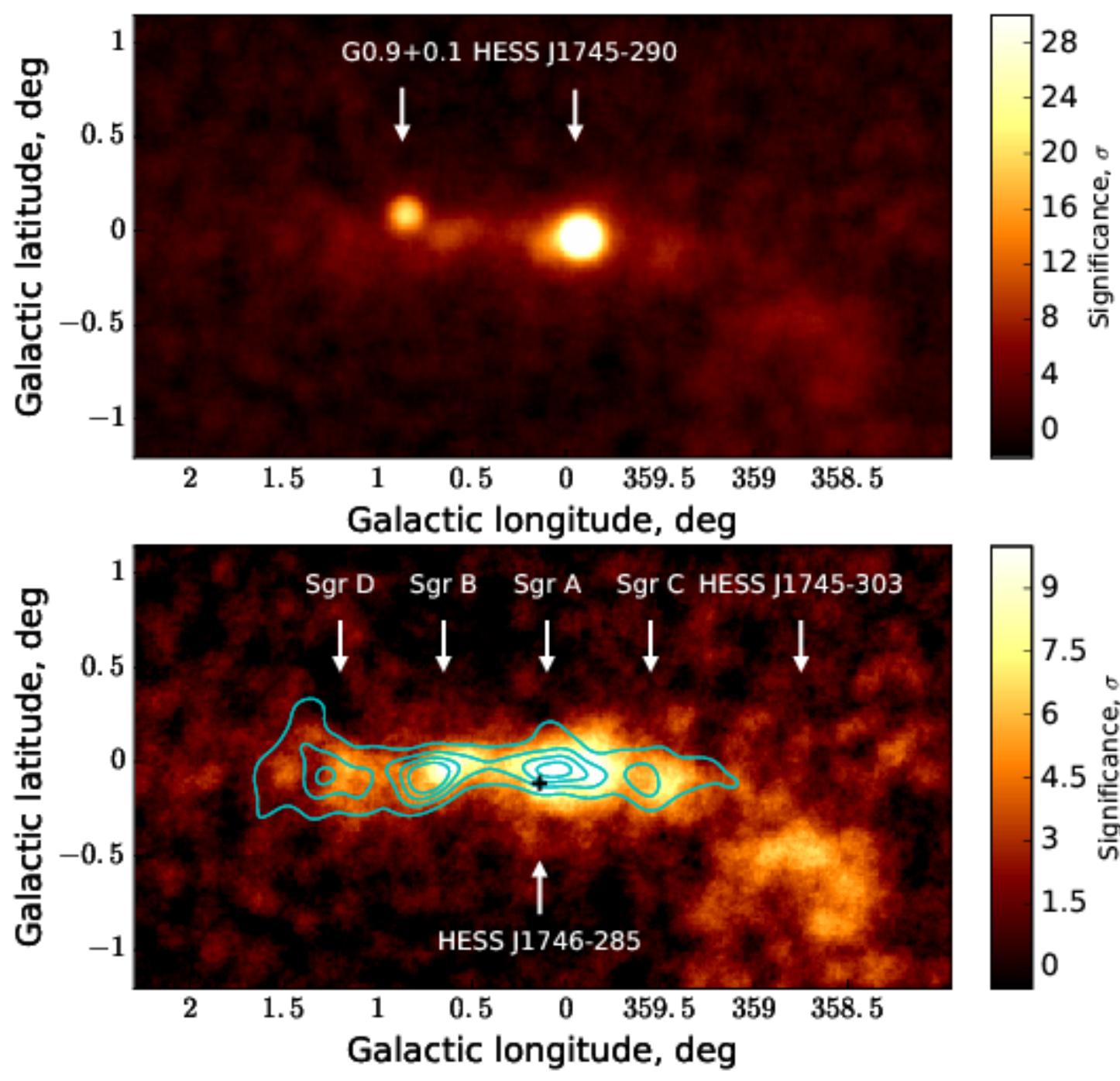

Figure 4: VHE $\gamma$-ray images of the galactic centre region. Top: $\gamma$-ray significance map. Bottom: Residual significance map after subtraction of the two point sources G0.9+0.1 and HESS J1745-290. The cyan contours indicate the density of molecular gas as traced by CS (smoothed with the H.E.S.S. PSF). Images reproduced from [6]

search Council, the Japan Society for the Promotion of Science and by the University of Amsterdam. We appreciate the excellent work of the technical support staff in Berlin, Durham, Hamburg, Heidelberg, Palaiseau, Paris, Saclay, and in Namibia in the construction and operation of the equipment. This work benefited from services provided by the H.E.S.S. Virtual Organisation, supported by the national resource providers of the EGI Federation.

\section{References}

[1] H.E.S.S. Collaboration, The H.E.S.S. Galactic plane survey, In preparation

[2] H.E.S.S. Collaboration, The population of TeV pulsar wind nebulae in the H.E.S.S. Galactic Plane Survey, Accepted for publication in A\&A [arXiv:1702.08280], 2017 


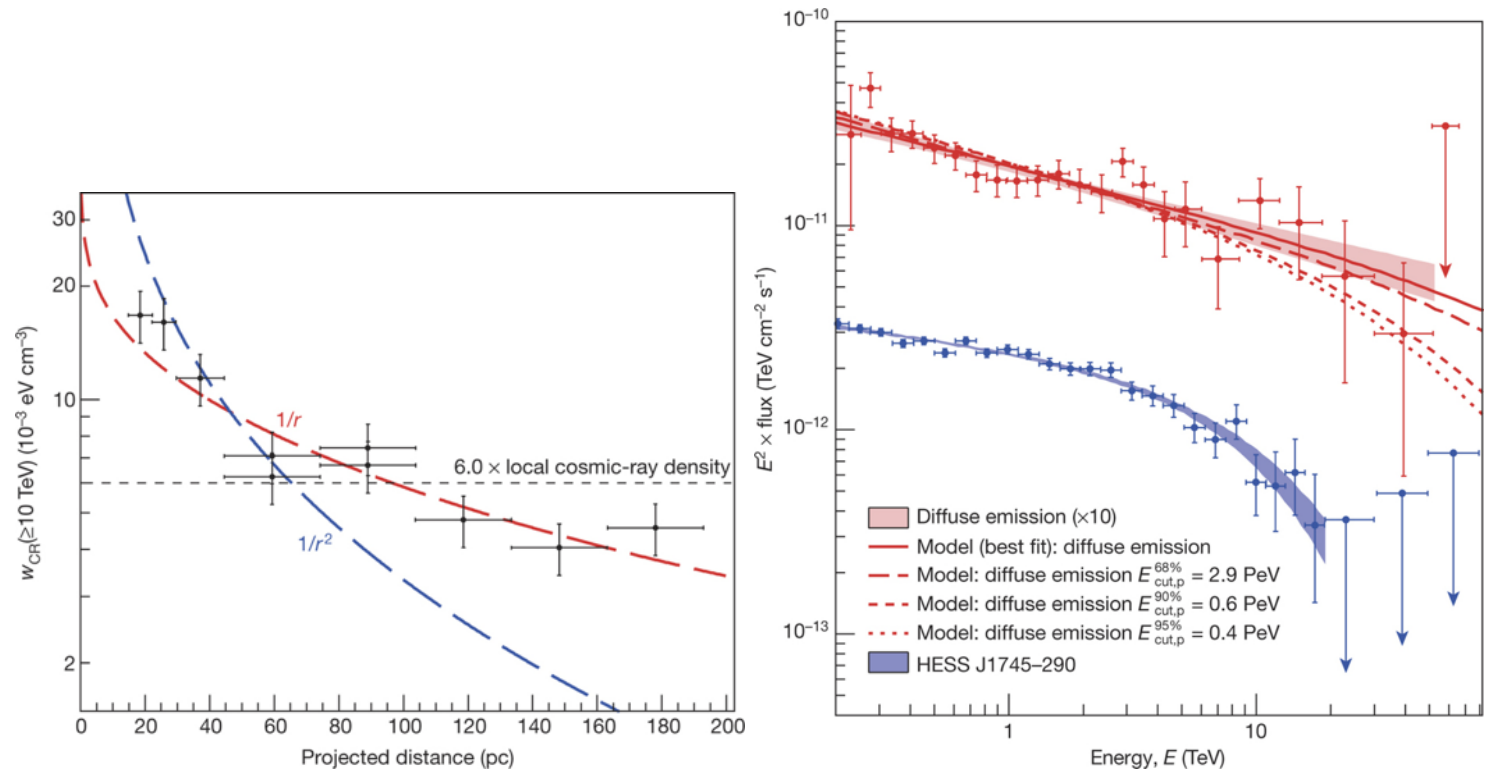

Figure 5: Distribution of cosmic ray density vs projected distance from the central source (left). Spectral energy distribution of the central source and surrounding diffuse emission, expected gamma-ray spectra are shown for several models of proton injection spectrum (right). Images reproduced from [7].

[3] H.E.S.S. Collaboration, Deeper H.E.S.S. Observations of Vela Junior (RX J0852.0-4622): Morphology Studies and Resolved Spectroscopy, Accepted for publication in A\&A [arXiv:1611.01863], 2016

[4] H.E.S.S. Collaboration, A search for new supernova remnant shells in the Galactic plane with H.E.S.S., Submitted to A\&A

[5] H.E.S.S. Collaboration, H.E.S.S. observations of RX J1713.7-3946 with improved angular and spectral resolution: Evidence for gamma-ray emission extending beyond the X-ray emitting shell, Accepted for publication in A\&A [arXiv:1609.08671], 2016

[6] H.E.S.S. Collaboration, Characterising the VHE diffuse emission in the central 200 parsecs of our Galaxy with H.E.S.S, Accepted for publication in A\&A [arXiv:1706.04535], 2017

[7] HESS Collaboration., Acceleration of petaelectronvolt protons in the Galactic Centre, Nature 531, 476, 2016 